\title{
Physicists in the Senate of the Kingdom of Italy: 1848-1943
}

\author{
Matteo Leone - Università di Torino; Centro di studi e ricerche "Enrico Fermi" \\ (Roma)-matteo.leone@unito.it
}

\begin{abstract}
The main results achieved by a project on the scientific and political activity of physicists and astronomers appointed by the King senators for life (1848-1943) are here discussed. This small, but significant, subset of senators for life was made up of physicists and astronomers who, "with eminent services or merits, had illustrated their homeland" (e.g. C. Matteucci and O.F. Mossotti) or, more frequently, who had been members of an Academy of Sciences for at least seven years (to this category belonged personalities such as G. Plana, V. Volterra, P. Blaserna and O.M. Corbino). The records preserved in the Senate of the Republic Historical Archives (Parliament Acts, Bills, Central Office reports and personal files) are a valuable source to understand the specific contributions of these "senators physicists" to the parliamentary work and the role that their "scientific" skills had on their parliamentary activity. The analysis of these documents shows how such contributions at times proved to be decisive for the legislative process. Also, this analysis highlights how the senators physicists' parliamentary activity spanned in the most diverse sectors of the country's life, such as education, research, infrastructures and energy issues. This project is the outcome of a collaboration between University of Turin, University of Genova and Senate of the Republic Historical Archives and was made possible by support from "Centro Fermi”" in Rome.
\end{abstract}

Keywords: Senators Physicists, Senate of the Kingdom, Kingdom of Italy.

\section{I "fisici senatori"}

La ricerca qui descritta è stata condotta in collaborazione con Nadia Robotti (Università di Genova) e l'Archivio Storico del Senato della Repubblica (nelle persone di Emilia Campochiaro ed Elisabetta Lantero) ed è stata finanziata dal "Centro Fermi" (Museo Storico della Fisica e Centro Studi e Ricerche "Enrico Fermi") nell'ambito del progetto interdisciplinare: HISTSEN - I fisici italiani tra ricerca scientifica e impegno civile: dal Congresso di Vienna all'avvento della Repubblica.

Gli obiettivi della ricerca sono la ricostruzione dell'impegno scientifico e politico dei fisici e degli astronomi che furono nominati Senatori del Regno nel periodo compreso tra il 1848 (Statuto Albertino) e il 1943 (caduta del Regime fascista) e la pubblicazione dei risultati principali in una monografia a cura della SIF in collaborazione con "Centro Fer- 
mi" e Archivio Storico del Senato dal titolo Scienza e impegno civile: i fisici al Senato (1848 - 1943) (autori M. Leone, N. Robotti).

Proseguimento ideale di un precedente lavoro sui fisici e il Risorgimento (Leone et al. 2011), l'impegno istituzionale dei fisici senatori è stato studiato attraverso fonti primarie quali atti parlamentari (Discussioni), fascicoli dei disegni di legge, fascicoli degli Uffici centrali (poi Commissioni), fascicoli personali e avvalendosi della banca dati I Senatori d'Italia a cura dell'Archivio Storico del Senato.

\subsection{Chi erano $i$ "fisici senatori” del Regno?}

Nel periodo oggetto di studio - 1848-1943 - si sono individuati sedici fisici senatori e cinque senatori astronomi, per un totale di ventuno senatori. Tre di questi senatori furono anche ministri di vari dicasteri, quali Pubblica istruzione, Economia nazionale e Marina.

I fisici senatori, che come tutti i senatori del Regno d'Italia erano nominati a vita dal $\mathrm{Re}$, appartenevano prevalentemente a due categorie di nomina tra le ventuno previste dallo Statuto Albertino:

- $\quad$ quindici fisici senatori appartenevano alla categoria 18, comprendente «i membri della Regia Accademia delle Scienze dopo sette anni di nomina»;

- quattro fisici senatori furono invece nominati nella categoria 20, ovvero per essere tra «coloro che con servizi o meriti eminenti avranno illustrata la Patria».

Un solo fisico senatore apparteneva invece alla categoria 5 «Ministri Segretari di Stato»e uno solo alla categoria 21 «persone che da tre anni pagano tremila lire d'imposizione diretta in ragione dei loro beni o della loro industria» (categoria, quest'ultima, alla quale appartenevano, ad esempio, buona parte dei senatori medici).

Visti nel complesso, i fisici senatori furono mediamente personaggi importanti dal punto di vista scientifico e noti a livello internazionale, come testimoniato dalla loro appartenenza ad accademie nazionali e internazionali (tra loro abbiamo figure quali Augusto Righi, che ottenne nominations per il premio Nobel in fisica per sedici anni consecutivi, e Guglielmo Marconi, Nobel per la fisica 1909).

Nel complesso, i fisici senatori si caratterizzarono anche per una relativamente "giovane" età di nomina. Ben dieci su ventuno furono infatti nominati ad un'età inferiore a 55 anni e addirittura nel caso di Marconi si attese il compimento dei 40 anni - limite previsto dallo Statuto - per nominarlo senatore.

I fisici senatori, inoltre, si caratterizzarono per una lunga permanenza in Senato, proprio perché solitamente nominati in età relativamente giovane. Ad esempio, Volterra fu senatore per 35 anni, Blaserna per 28, Marconi per 23 e Corbino per 17. Un'eccezione fu quella di Galileo Ferraris che, nominato a 49 anni in Senato (da lui definito «il più grande laboratorio della Patria»), morì l'anno dopo.

Per quanto riguarda la distribuzione nel tempo, i fisici senatori furono numericamente abbastanza rilevanti. Per un lungo periodo nel Regno di Sardegna (dal 1848 al 1859) l'unico rappresentante di questa categoria fu l'astronomo Giovanni Plana. Negli anni che portano all'Unità d'Italia ne furono nominati altri quattro: Carlo Matteucci, Ernesto Capocci, Annibale De Gasparis e Ottaviano Mossotti. Alle soglie dell'Unità vi erano quindi 
5 fisici senatori su un totale di circa 150 senatori che allora sedevano in Senato. Il periodo liberale (1861-1922) fu quello che vide il maggior numero di nomine: Luigi Palmieri, Giovanni Cantoni, Giovanni Schiaparelli, Pietro Blaserna, Galileo Ferraris, Emanuele Fergola, Antonio Pacinotti, Augusto Righi, Vito Volterra, Giovanni Celoria, Pasquale Leonardi Cattolica, Guglielmo Marconi, Guglielmo Mengarini, Orso Mario Corbino. Solamente due, infine, i fisici senatori nominati in epoca fascista (1922-1943): Antonio Garbasso e Luigi De Marchi. L'anno di maggior "concentrazione" fu il 1910, nel quale sedevano in contemporanea in Senato otto fisici senatori (Schiaparelli, Blaserna, Fergola, Pacinotti, Righi, Volterra, Celoria e Leonardi) su un totale di circa 300 senatori presenti in Aula.

L'attività legislativa dei fisici senatori fu molto intensa salvo pochissime eccezioni. A primeggiare nell'attività parlamentare furono Blaserna con novantacinque interventi, Corbino con sessantasei, Leonardi Cattolica con quaranta, Volterra con diciassette, Mengarini con quattordici, Righi con tredici.

Dagli atti parlamentari emergono interventi di altissimo livello, che spesso attirano l'attenzione dell'Aula: i testi degli interventi sono frequentemente accompagnati da espressioni quali «(Acclamazioni)», «(Applausi)», «(Congratulazioni)». Pur con una "densità" variabile nel corso dei decenni, i temi sui quali si è concentrato l'interesse e il lavoro dei fisici senatori possono essere suddivisi in quattro aree:

- assetto istituzionale del Paese;

- istruzione;

- economia e infrastrutture;

- ricerca.

Per ciascuna di queste aree vedremo nel seguito esempi di interventi particolarmente significativi, che danno la misura di quanto il contributo dei fisici senatori all'attività legislativa del Senato fu importante e ricco di conseguenze.

\section{Assetto istituzionale del Paese}

L'analisi degli atti parlamentari evidenzia come i fisici senatori ebbero un ruolo importante in diversi passaggi cruciali della storia d'Italia. Tra questi passaggi cruciali, sicuramente vanno annoverati i seguenti:

- $\quad$ il processo che porta all'Unità d'Italia e, in particolare, il trattato di cessione di Nizza e Savoia alla Francia (1860), la proclamazione di Vittorio Emanuele II come primo Re d'Italia (1861) e la questione romana (1861);

- $\quad$ in materia di sistemi elettorali, l'introduzione dello scrutinio di lista (1882);

- $\quad$ per quanto concerne la pubblica amministrazione, la questione dei monopoli di Stato (1896) e il riordino del sistema tributario e amministrativo (1922); 
- infine, in materia di politica estera, i fisici senatori intervengono sulle colonie italiane d'Africa (1908) e su diversi temi relativi alla Grande Guerra (cooperazione economica e, più in generale, rapporto tra scienza e guerra).

Uno degli esempi maggiormente significativi di coinvolgimento dei fisici senatori nel dibattito legislativo sull'assetto istituzionale del Paese è rappresentato dal progetto di legge con cui Vittorio Emanuele II assume il titolo di Re d'Italia.

\subsection{Matteucci e Vittorio Emanuele II Re d'Italia}

Il 21 febbraio 1861, Vittorio Emanuele II, ancora Re di Sardegna, autorizzava la presentazione in Senato da parte di Cavour di tale progetto. Come da regolamento parlamentare, il progetto veniva sottoposto all'esame dell'Ufficio centrale del Senato. ${ }^{1}$ Tre giorni dopo, l'Ufficio centrale riferiva all'Assemblea il proprio parere. Relatore dell'Ufficio centrale era Carlo Matteucci (nominato senatore il 18 marzo 1860).

Nella propria relazione il fisico senatore argomentava il proprio parere positivo sul progetto in esame:

Il titolo di Re d'Italia pone in atto il concetto intero della volontà nazionale, cancella i simboli delle nostre interne divisioni, è per l'animo d'ogni italiano un pegno di grandezza e di unione, accresce l'autorità del Governo del Re nei Consessi europei ed offre alle grandi potenze, in mezzo alle quali il regno d'Italia prende posto, degna occasione per accettare il risorgimento politico di un popolo che ha tanto contribuito alla civiltà universale (Matteucci 1861a, p. 5).

Matteucci suggeriva tuttavia un'ulteriore integrazione al progetto di legge, in relazione all'intestazione degli atti legislativi, «di cui sembra non possa essere contestata la ragione e la convenienza e per la quale il fatto memorando ed il principio giuridico della novella monarchia siano ognora presenti al popolo italiano e congiunti al nome de' suoi Re». Tale proposta di integrazione consisteva nell'intestazione degli atti del Governo, e di ogni altro atto intitolato in nome del Re, con la formula «Per Provvidenza Divina, per voto della Nazione».

Dal fascicolo del disegno di legge emerge come tale emendamento al progetto originale sia stato frutto di un'apposita integrazione rispetto a una prima stesura della relazione dell'Ufficio centrale, forse con l'obiettivo di far sì che il provvedimento incontrasse il consenso maggiore possibile (Fig. 1):

La Provvidenza Divina che mai si rivela meglio nella sua bontà e nella sua giustizia che quando muove e dirige la volontà dei popoli a riconquistare dritti o manomessi o perduti; la virtù, la concordia e la perseveranza italiana che la mirabile opera hanno

\footnotetext{
${ }^{1}$ Il regolamento parlamentare prevedeva che l'assemblea si dividesse per estrazione a sorte in cinque Uffici, rinnovati ogni due mesi, e che i disegni di legge fossero esaminati da tali Uffici. I cinque Uffici avevano il compito di esaminare le proposte di legge che, una volta esaminate, dovevano essere trasmesse ad un Ufficio centrale composto dai commissari nominati da ciascun ufficio, oppure ad una commissione speciale, o infine ad una delle commissioni permanenti competenti per materia. L'Ufficio centrale o la commissione, al termine dell'esame, nominava un relatore con il compito di riferire all'Assemblea.
} 
compito debbono associarsi al nome del Re, siccome la ragione più cara e la forza più salda del Regno (Archivio della Camera Regia, Disegni e proposte di legge e incarti delle commissioni (1848-1943), Minuta della Relazione dell’Ufficio centrale, c. 9bis).

Nella seduta del Senato del successivo 26 febbraio il Governo, nella persona del ministro di Grazia e giustizia Giovanni Battista Cassinis, pur approvando l'integrazione di Matteucci in quanto "tributo di riconoscenza all'Ente Supremo», suggeriva che tale norma non fosse contenuta nel disegno di legge in oggetto, ma «nelle disposizioni preliminari del Codice civile $[\ldots]$ od in altra legge apposita e speciale».

Nella medesima seduta Matteucci prendeva atto «che quel secondo articolo sarà formulato in un progetto speciale di legge», si dichiarava «appagato» e consentiva che la legge fosse limitata al primo articolo proposto dal Governo (Discussione sul progetto di legge 1861, p. 29). Il progetto, approvato all'unanimità sotto forma di articolo unico, diventava così la legge 17 marzo 1861 n. 4671, la legge con cui l'VIII legislatura proclamava il Regno d'Italia.

Già il 18 marzo il Ministro Cassinis presentava, come promesso, il progetto di legge di intitolazione degli atti di Governo. Ancora una volta era Matteucci relatore dell'Ufficio centrale. Matteucci motivava il ricorso a una legge apposita con l'argomento che «aggiungere un secondo articolo avrebbe diminuita in qualche modo la semplicità di quella prima legge, e non lasciato dominare interamente il grande fatto che essa esprime» (Matteucci 1861 b, p. 71).

Matteucci appoggiava quindi la proposta del ministro secondo cui ogni atto di Governo intitolato in nome del Re debba essere intestato, similarmente alla proposta originale, con la formula «Per Grazia di Dio e per Volontà della Nazione».

Entrambe le Camere approvavano il provvedimento e il 21 aprile 1861 veniva emanata la legge sull'intitolazione degli atti di Governo: la legge n. 1 del Regno d'Italia!

E così, dietro due leggi di altissimo valore simbolico, la legge con cui veniva proclamato il Regno d'Italia e la legge n. 1 emanata dal nuovo Regno, troviamo lo stesso nome, quello di un fisico senatore. 


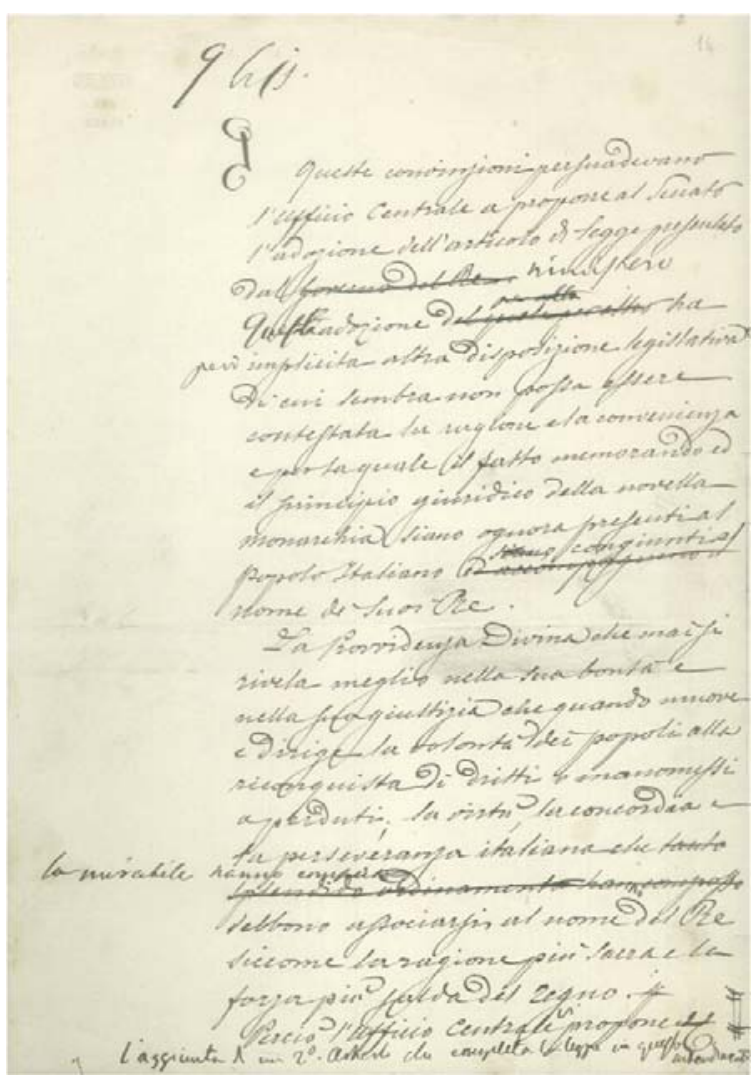

Fig. 1. Integrazione, nella relazione di Matteucci, con cui si motiva l'introduzione di un secondo articolo facente riferimento alla "Provvidenza Divina" e al voto della Nazione come fondamento del titolo di "Re d'Italia".

\section{Istruzione}

Anche sul fronte dell'istruzione gli atti parlamentari evidenziano un grande attivismo dei fisici senatori.

Per quanto riguarda l'istruzione elementare e media, si registrano interventi sulla riforma della legge Casati (1859), sulle pensioni e lo stato giuridico degli insegnanti, sul progetto di Ginnasio liceo moderno (1911), sul problema dell'analfabetismo (1921) e sulla riforma Gentile (1923).

In merito all'istruzione universitaria, invece, vanno ricordati il progetto di Matteucci di riordinamento dell'istruzione superiore (1861), gli interventi sul progetto di riforma Coppino (1886) e ancora una volta quelli sulla riforma Gentile.

Emblematica dell'attenzione dei fisici senatori per le questioni legate all'istruzione, è l'azione di Orso Mario Corbino sul problema dell'analfabetismo. 


\subsection{Corbino e l'opera contro l'analfabetismo}

Nel periodo successivo all'Unità d'Italia si succedettero vari tentativi per il recupero degli adulti analfabeti o semianalfabeti, soprattutto a opera di istituzioni private o religiose. Tali tentativi si intensificarono subito dopo la prima guerra mondiale ad opera dello Stato, come dimostrato dal varo, il 2 settembre 1919, di un "Ente nazionale per l'istruzione degli adulti analfabeti". Il ministro affida a tale ente «le funzioni allo Stato devolute nei riguardi della istruzione degli adulti analfabeti e della diffusione della istruzione elementare nei centri minori di popolazione» (art. 5).

Orso Mario Corbino (nominato Senatore il 3 ottobre 1920, ministro della Pubblica istruzione nel primo governo Bonomi, dal 4 luglio 1921 al 22 febbraio 1922), osserverà in Senato nel 1921:

l'esperienza non dimostrò il funzionamento dell'Ente corrispondente ai fini che ne avevano ispirata l'istituzione [...]. Difatti l'Ente ebbe il tempo di nascere, di vivere male e di morire (Svolgimento dell'interpellanza del senatore Del Giudice 1923, p. 1240).

Da ministro della Pubblica istruzione Corbino, con l'obiettivo di potenziare i mezzi per abbattere l'analfabetismo, decise di istituire, con decreto legge del 28 agosto 1921, una nuova incarnazione dell'ente, denominato "Opera contro l'analfabetismo" per «combattere l'analfabetismo degli adulti e della popolazione sparsa e fluttuante, a cominciare dalle regioni che hanno una più alta percentuale di analfabeti» (Fig. 2).

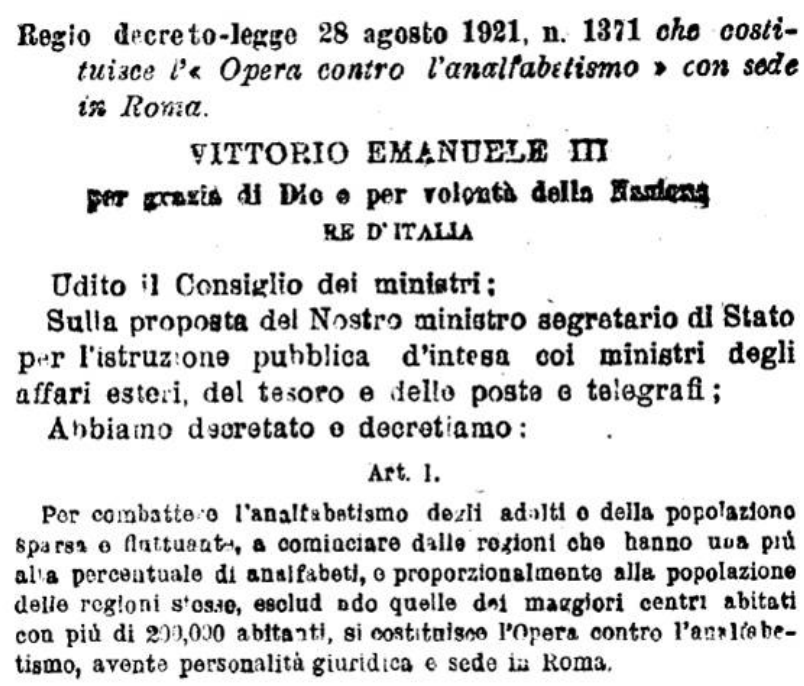

Fig. 2. Decreto di costituzione dell'“'Opera contro l'analfabetismo".

Nel suo discorso in Senato, il 28 giugno 1922, Corbino (non più ministro) espliciterà obiettivi e strategie dell'“Opera contro l'analfabetismo". Per Corbino: 
Anzitutto occorre seguire i bambini che hanno già lasciata la scuola obbligatoria $\mathrm{e}$ troppo presto vengono allontanati dal contatto con istituti culturali ritornando così in un certo senso a costituire una categoria speciale di post-analfabeti. [È] necessario far sorgere scuole accanto ai centri industriali o agricoli isolati perché o nelle giornate festive o nelle ore serali si impartissero complementi di istruzione. [Inoltre] occorre far sorgere scuole festive per tutti gli operai e scuole serali e festive per gli adulti. Insomma, tutti compiti ai quali lo Stato non provvede (Seguito della discussione di "Stati di previsione" 1923, p. 3145).

A differenza del vecchio ente, totalmente in mano allo Stato, l'Opera si avvaleva anche di privati, ai quali era delegata l'azione contro l'analfabetismo degli adulti. L'articolo 2 del predetto decreto enunciava l'organigramma dell'Opera ed elencava le associazioni delegate a tale azione.

A chi in Senato obiettava che l'istituzione dell'Opera non rivestirebbe caratteri d'urgenza, Corbino così rispondeva il 12 dicembre 1921, dopo aver osservato che lo strumento del decreto-legge aveva consentito la rapida istituzione di circa 2.500 scuole:

Se col guadagno di un anno otterrò che cinquantamila adulti analfabeti d'Italia riescano fra un anno a togliersi questa macchia che offusca la loro bella intelligenza, non dovrò certamente pentirmi (Svolgimento dell'interpellanza del senatore Del Giudice 1923, p. 1242).

Nel proprio discorso del 12 dicembre 1921 Corbino faceva riferimento al suffragio universale, introdotto con la legge 16 dicembre 1918, che dichiarava elettori tutti i cittadini maschi di almeno 21 anni di età. ${ }^{2}$

\begin{abstract}
Abbiamo, con il suffragio universale (concesso anche agli analfabeti), abbassato l'esercizio della sovranità popolare al livello della ignoranza più umiliante, piuttosto che elevare la preparazione intellettuale delle masse all'altezza necessaria per il decoroso esercizio di quella sovranità. Abbiamo dato all'Italia ampiezza, coscienza e dignità di grande Nazione, senza aver cura che la media degli analfabeti fra i cittadini italiani si stacchi molto dal livello raggiunto presso i paesi balcanici. Quest'onta deve cessare: per ragioni di interesse e di sentimento (Svolgimento dell'interpellanza del senatore Del Giudice 1923, p. 1242).
\end{abstract}

A proposito delle ragioni "di sentimento", così argomentava Corbino:

Non dobbiamo più oltre tollerare che dei nostri fratelli vivano ancora con questa benda oltraggiosa che ne sbarra la fervida intelligenza e ne paralizza le mirabili forze. Dobbiamo convincerci che questo problema è più urgente di ogni altro, anche di quelli relativi alle scuole medie e superiori, dove si affollano anche troppi studenti, al di là dei bisogni effettivi del paese (Svolgimento dell'interpellanza del senatore Del Giudice 1923, p. 1243).

\footnotetext{
${ }^{2}$ Già il Testo Unico del 26 giugno 1913 aveva esteso il diritto di voto ai cittadini maschi di oltre 30 anni, anche se analfabeti, ed ai cittadini di età compresa tra 21 e 30 anni che sapessero leggere e scrivere, o fossero in possesso dei requisiti fissati dalle precedenti leggi o avessero compiuto il servizio militare.
} 
Nel suo discorso in Senato del 28 giugno 1922 Corbino illustrava i primi risultati conseguiti con l'istituzione dell'Opera:

Posso dire che fino al tempo in cui io mi trovavo al Ministero già 2.000 di queste
scuole speciali erano state istituite, e che molte diecine di migliaia le frequentavano.
Non è a dirsi poi l'entusiasmo con cui questa iniziativa è stata accolta dovunque, e
soprattutto nei miei paesi della Sicilia. A questo proposito potrei citare alcuni esem-
pi veramente commoventi. In alcuni comuni l'intera popolazione andava ad acco-
gliere la suppellettile scolastica con dimostrazioni festose. Vi sono stati comuni che
hanno creduto di incoraggiare l'istituzione con premi speciali a favore di alunni che
più si distinguevano; comuni (e questo è veramente sintomatico) che pur avendo le
proprie scuole si rivolgevano all'Associazione delegata dicendo: vi diamo i mezzi
purché ci istituiate delle scuole con i vostri metodi.
Orbene, quale è il segreto di questi metodi? I maestri sono pagati per quello che ren-
dono; non c'è stabilità di impiego (Seguito della discussione di "Stati di previsione"
1923, p. 3145).

Risultati incoraggianti, che inducevano Corbino, nella medesima seduta del Senato del 1922, ad esprimersi con grande soddisfazione su quanto realizzato con l'Opera nella battaglia contro l'analfabetismo. Per il fisico senatore, infatti, l'istituzione dell'Opera contro l'analfabetismo «è il solo atto del mio Ministero, del quale avrò sempre a lodarmi».

\section{Economia e infrastrutture}

Numerosi gli interventi anche sul fronte dell'economia e delle infrastrutture. Tra le infrastrutture la cui realizzazione vide il coinvolgimento diretto dei fisici senatori vi furono le linee telegrafiche (in particolare la prima linea telegrafica del Regno sardo e la linea telegrafica da Torino al confine lombardo, tra il 1851 e il 1852), la propulsione idropneumatica come metodo per il trasporto ferroviario (1854), la costruzione di una stazione radiotelegrafica "ultrapotente" ad opera di Marconi (1903) e la nascita della posta aerea (1917).

Altro fronte attivo fu quello delle risorse energetiche e minerarie, attraverso questioni quali la ricerca e l'uso di sostanze radioattive (1919), la derivazione delle acque pubbliche (1920), l'elettrificazione delle ferrovie (1920), lo sfruttamento della lignite (1923) e l'unificazione della legislazione mineraria (1925).

\subsection{Plana e la propulsione idropneumatica}

Il progetto della linea ferroviaria tra Torino-Genova, realizzata tra il 1846 e il 1853, mette in luce un singolare ed interessante caso di rapporto tra Stato e ricerca scientificotecnologica.

L'ostacolo maggiore al completamento della linea era la realizzazione della galleria dei Giovi, caratterizzata dal cosiddetto "piano inclinato dei Giovi”, lungo circa 10 km e di pendenza marcata, che in un tratto raggiunge il 3,5\%. La realizzazione della galleria dei Giovi, oltre che per il suo interesse economico e strategico, rappresentava quindi una non 
banale sfida tecnologica. La realizzazione di tale infrastruttura è tuttavia significativa anche sotto un altro profilo, trattandosi infatti di un caso nel quale lo Stato non solo finanziò un'infrastruttura ma anche il progetto per una ricerca scientifico-tecnologica finalizzata allo studio della miglior tecnologia da utilizzarsi per il trasporto, soprattutto delle merci, lungo la linea Torino-Genova. E al dibattito che si tenne in Senato su questo progetto partecipò attivamente anche l'astronomo Giovanni Plana (nominato senatore il 3 aprile 1848).

Una Commissione speciale, nominata nel dicembre 1851, fu incaricata «di studiare il miglior sistema di locomozione applicabile ai Giovi». Si valutarono tre diverse soluzioni per permettere ai treni di affrontare il difficile piano inclinato:

$1^{\circ}$ Quello delle locomotive che si muovono in virtù dell'aderenza delle loro ruote sui rails;

$2^{\circ}$ Quello della fune avvolta a due tamburi come a Blackwol che era lunga 6087 metri, oppure come a Liegi a Plan Meliné, da Liverpool a Glasgow, ad Aix la Chapelle lunghi circa di 2000 metri, ove la fune è a movimento continuo;

$3^{\circ}$ Quello del tubo del sistema atmosferico, collocato nel mezzo e lungo tutto la via, fessurato dal lato superiore per il passaggio del braccio rimorchiatore che si muove collo stantuffo spintovi dalla differenza di tensione dell'aria racchiusavi, la fessura fermandosi con apposite valvole (Discussione generale del progetto 1870b, p. 2080).

Il primo metodo, quello che poi sarà effettivamente adottato, era quello delle locomotive, le quali, però, all'epoca davano risultati non del tutto soddisfacenti, richiedendo una spesa molto elevata per una quantità relativamente limitata di merci trasportate. Al 1854, sul piano dei Giovi si trasportavano infatti giornalmente 600 tonnellate di merci (passeggeri compresi), per un costo di 800 lire di combustibile al giorno, corrispondenti a 300.000 lire all'anno.

Il secondo metodo prevedeva l'utilizzo di una trazione a fune comandata da una stazione fissa sui Giovi ospitante numerose macchine a vapore. L'uso di questa tecnica, tuttavia, fu presto abbandonato anche sulle linee citate per le perdite di tempo che comportava e per il contestuale miglioramento tecnologico delle locomotive in termini di peso e di potenza.

Il terzo metodo, utilizzato negli anni Quaranta dell'Ottocento nel Regno Unito e in Francia (Fig. 2), contemplava l'utilizzo di una propulsione idropneumatica, ovvero far sì che a spingere il convoglio fosse un braccio rimorchiatore collegato a uno stantuffo situato in un tubo, posto tra $\mathrm{i}$ binari, ai cui estremi si produceva una differenza di pressione dell'aria tramite l'acqua proveniente da un bacino.

Il 26 settembre 1853 gli ingegneri Sebastiano Grandis, Severino Grattoni e Germain Sommeillier facevano "domanda del privilegio" (brevetto) di un sistema di locomozione, eventualmente applicabile al piano inclinato dei Giovi, secondo il $3^{\circ}$ metodo (Fig. 3), ovvero di un

Sistema idraulico per comprimere e rarefare i fluidi elastici coll'azione diretta dell'acqua mediante il quale la forza motrice di questa si trasforma in forza elastica, 
agisce in qualunque luogo o direzione, riesce trasportabile, e diventa infine applicabile dovunque ed in genere alla locomozione, alle arti e all'industria (Discussione generale del progetto 1870 b, p. 2090).

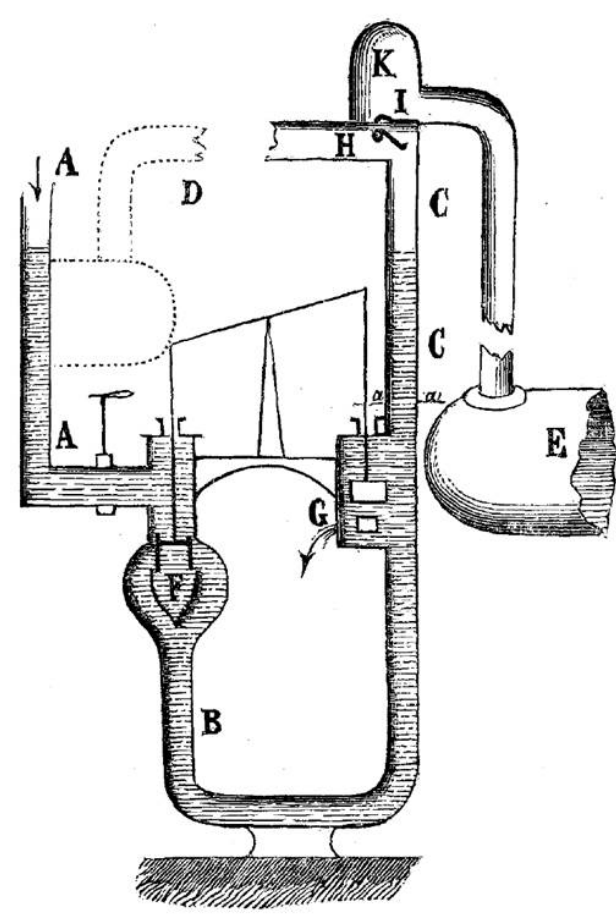

Fig. 3. Schema del sistema idraulico presentato da Grandis, Grattoni e Sommeiler (Discussione generale del progetto 1870 b, p. 2090).

Il 20 novembre 1853 si aveva il parere favorevole dell'Accademia delle Scienze di Torino e il successivo 15 gennaio 1854 veniva emanato il decreto di privilegio. Il 20 febbraio 1854 si teneva la solenne inaugurazione della ferrovia.

Facendo seguito al brevetto dei tre ingegneri, il 10 aprile dello stesso anno Cavour presentava alla Camera il disegno di legge per l'applicazione del sistema di propulsione idropneumatica nel passaggio dei Giovi. Il disegno di legge consisteva di due articoli, il primo dei quali chiedeva l'approvazione di una convenzione tra il Ministero delle finanze e i tre ingegneri, mentre il secondo stanziava la somma di 120 mila lire «per l'effettuazione degli esperimenti». Il Ministero stanziava una somma, quindi, non per la diretta realizzazione di un'infrastruttura, bensì per l'effettuazione di esperimenti atti a validarne l'efficacia. In particolare, la convenzione stabiliva che:

Prima d'intraprendere lo stabilimento definitivo del sistema, si farà un'esperienza tendente a dimostrare l'efficacia della macchina idropneumatica, e del tubo propulsore. 
A questo fine si costruirà un apparecchio completo con una lunghezza di tubo propulsore di duecento metri almeno. L'esperimento avrà luogo sopra un tratto di ferrovia provvisoria, da stabilirsi in sito conveniente lateralmente alla strada del Governo. Le spese dell'esperimento non potranno sorpassare la somma di ottanta a novanta mila lire; esse saranno interamente a carico dell'Amministrazione; ma in caso di non favorevole riuscita, gl'ingegneri Grattoni, Grandis e Sommeiller dovranno rimborsare al Governo le spese che gli avranno cagionate (Archivio della Camera regia, Disegni e proposte di legge e incarti delle commissioni (1848-1943), 308, 310-334 cc., vol. X).

L'Ufficio centrale del Senato, nella sua relazione del successivo 4 luglio, esprimeva parere favorevole al progetto poiché «sostanzialmente è tutelato l'interesse dello Stato». Infatti, «colla semplice anticipazione d'una ben discreta somma, il cui rimborso è assicurato pel caso di non successo» e «avverandosi le concepite speranze», si otteneva «una considerevole economia nella spesa di propulsione dei convogli di merci lungo il piano dei Giovi» e di poter superare «anche in altre località difficoltà giudicate sin qui insuperabili» (Mosca 1869, p. 1348).

La discussione in Senato si teneva il successivo 8 luglio e vedeva anche la partecipazione di Plana, il quale criticava il citato articolo della convenzione, reputato ingeneroso nei confronti dei tre ingegneri. Ad avviso di Plana,

Mi pare che sarebbe atto generoso e giusto quello di non adottare l'articolo 4. Si facciano gli esperimenti, e siano liberi gli inventori da qualsivoglia multa ove non sortissero l'effetto utile che se ne propongono.

La dignità del Governo consiglia di concedere quella somma di 120 mila lire senza sottoporre gli inventori alla condizione della restituzione in verun caso.

L'accettazione di quelle condizioni prova che gl'inventori hanno piena fiducia nell'esito, e l'esporsi a tanto rischio è atto lodevole e ben degno di essere accolto senza il peso di quelle enormi misure. [...] [Queste] persone ci mettono la loro riputazione che val ben più di 120 mila lire (Discussione ed approvazione del progetto di legge 1870a, pp. 333-334).

Per lo scienziato Plana, quindi, non era "dignitoso" che il Governo chiedesse agli ingegneri di rispondere in proprio dell'eventuale fallimento dell'esperimento. In risposta a Plana, il Ministro Paleocapa osservava che «l'obbligo di questo eventuale rimborso venne di buon accordo pattuito tra il Governo e gl'ingegneri Grandis, Grattoni e Sommeiller» in seguito alle pressioni della Camera dei deputati, la quale aveva «desiderato che s'imponesse loro l'obbligo di guarentire in modo legale l'esecuzione di tutte le obbligazioni da essi assunte».

Al termine della discussione, il progetto veniva comunque approvato come proposto dall'Ufficio centrale e diventa la legge 20 luglio 1854.

Di fatto, ragioni economiche indurranno a non utilizzare il metodo idropneumatico per la propulsione nel piano inclinato dei Giovi. Non ci fu quindi bisogno di mettere in gioco la reputazione dei tre ingegneri, reputazione che, come osservava Plana, «val ben più di 120.000 lire». 
Tale metodo troverà invece, di lì a poco, un'importantissima applicazione nella realizzazione della galleria del Frejus, progettata dagli stessi Grandis, Grattoni e Sommeiller. Durante i lavori si fece infatti ampiamente uso del metodo idropneumatico, non per vincere le forti pendenze, ma per muovere le macchine perforatrici (al fine di realizzare i fori di mina dove collocare le cariche esplosive) e per ventilare i lavori nella galleria. I lavori di scavo del Frejus, iniziati nel 1857, si conclusero nel 1870 e il traforo fu inaugurato l'anno successivo. Una delle più grandi infrastrutture realizzate durante il Regno sardo vedeva finalmente la luce. Un'opera realizzata con l'aiuto di un metodo il cui studio era stato incoraggiato dal Senato e, in particolare, da Plana.

\section{Ricerca}

La maggioranza degli interventi sulla ricerca dei fisici senatori riguardano questioni relative allo studio dell'ambiente, ad esempio in ambito meteorologico, e strategie per far fronte a tragedie naturali, quali i terremoti disastrosi che si verificarono in Calabria e Sicilia nel 1905 e nel 1908.

\subsection{Blaserna e il terremoto di Messina}

Epocale fu il terremoto di Messina del 28 dicembre 1908. Si trattò della più grave catastrofe naturale nel continente europeo per numero di vittime: il $42 \%$ della popolazione di Messina e il $21 \%$ di quella di Reggio Calabria, per un totale di circa 80.000 morti e un danno economico complessivo stimato in circa 600 milioni di lire, una cifra superiore all'ammontare complessivo dell'interesse sul debito pubblico del periodo 1907-1912.

La risposta delle istituzioni all'emergenza ebbe inizio con la legge n. 12 del 12 gennaio 1909 , con la quale furono stabilite le prime assegnazioni per bisogni ed opere urgenti.

L'art. 7 della legge n. 12 prevedeva anche la costituzione di una «speciale commissione consultiva da nominarsi con decreto Reale» atta ad individuare «le norme tecniche ed igieniche obbligatorie per le riparazioni, ricostruzioni e costruzioni nuove degli edifici pubblici e degli edifici privati, nei Comuni colpiti dal terremoto del 28 dicembre 1908 o da altri precedenti».

La commissione reale, che era stata caldeggiata pochi giorni prima dal físico Pietro Blaserna (nominato senatore il 4 dicembre 1890) in una lettera al presidente del Consiglio Giolitti, con la quale si candidava anche a presiederla, veniva costituita con il regio decreto del 15 gennaio 1909. Lo stesso Blaserna veniva designato presidente della commissione.

Il lavoro della commissione di Blaserna fu oggetto di accesa discussione in Senato il 21 maggio 1909 a seguito dell'interpellanza di un senatore di Messina (Todaro) al ministro dell'istruzione pubblica (Rava) «per sapere in che modo intenda il Governo ristabilire l’Università di Messina».

Nella propria replica, il Ministro Rava faceva riferimento alla commissione di Blaserna: 
La costruzione della Università corona l'opera di ricostruzione: senza le case non ci sarà la gente, e, senza la gente e le case, non si avranno studenti, professori, le condizioni di vita necessarie. Ed è un problema subordinato alle indagini della Commissione scientifica che deve suggerire i modi e il luogo per ricostruire la città. Questo studio sarà pronto tra poche settimane, perché ne è garanzia l'alacre operosità dell'illustre presidente della Commissione, il senatore Blaserna: quando questa avrà espresso le sue ragioni e proposte scientifiche, sapremo anche dove il nuovo edifizio potrà sorgere (Svolgimento dell'interpellanza del senatore Todaro 1909, p. 127).

Nella propria contro-replica, il senatore Todaro ribadiva la richiesta di un'immediata ricostruzione degli edifici universitari e sosteneva l'inopportunità di attendere la conclusione dei lavori della Commissione Blaserna.

Le parole del senatore Todaro suscitavano l'immediata, piccata, replica del fisico senatore:

Signori, non era veramente mia intenzione di prendere la parola in questa occasione. Ma il collega Todaro si è rivolto a me ed ha fatto sapere al Senato che, se si aspetterà la fine degli studi della Commissione da me presieduta, la ricostruzione dell'Università di Messina avverrà non si sa quando. Tengo quindi a dichiarare nel modo più formale che la Commissione per lo studio delle zone sismiche, che io ho l'onore di presiedere, tra breve, forse tra un mese, presenterà le sue conclusioni ed avrà così terminato il suo lavoro. (Bene) (Svolgimento dell'interpellanza del senatore Todaro 1909, p. 130).

Subito dopo la replica di Blaserna, interveniva nuovamente il ministro Rava per plaudire al lavoro del fisico senatore e della sua Commissione.

\begin{abstract}
Ringrazio prima di ogni altra cosa l'illustre senatore Blaserna che con l'autorità del suo nome e dei suoi studi ha posto la questione sul suo vero terreno, ricordando come compito della Commissione scientifica da lui presieduta sia quello di determinare la zona dove possa essere ricostruita l'Università. Tutti sanno con che cura il senatore Blaserna ed i suoi illustri colleghi si sono dati alla risoluzione di questo problema e quindi io attendo con piena fiducia le non lontane certo conclusioni dei loro lavori (Svolgimento dell'interpellanza del senatore Todaro 1909, p. 131).
\end{abstract}

Come anticipato da Blaserna in Senato, la sua Commissione concludeva effettivamente i lavori a fine giugno del 1909.

Le conclusioni, di lì a poco incorporate in un decreto reale (Regio decreto n. 542, 15 luglio 1909), erano chiare ed apparentemente "sorprendenti": Messina, pur essendo situata sopra terreno alluvionale estremamente instabile, in un'area oltretutto soggetta a forti scosse sismiche, doveva essere ricostruita lì dove era nata (Fig. 4).

Quelle stesse leggi economiche e geografiche che avevano fatto sì che in tale sito fosse eretta, inducevano la commissione Blaserna a concludere che era opportuno mantenere la città nella medesima zona d'impianto e nelle immediate vicinanze del porto, fatto salvo il rispetto di requisiti di sicurezza da parte dei nuovi edifici e un'opportuna distanza minima dalla linea di costa. 
Il lavoro della commissione era concluso e il processo di ricostruzione poteva partire. Tuttavia, questo non rappresentò la parola fine nel coinvolgimento del físico senatore. Anche dopo ultimati i lavori della commissione, Blaserna continuerà infatti ad occuparsi attivamente in Senato delle tragiche conseguenze del terremoto di Messina.

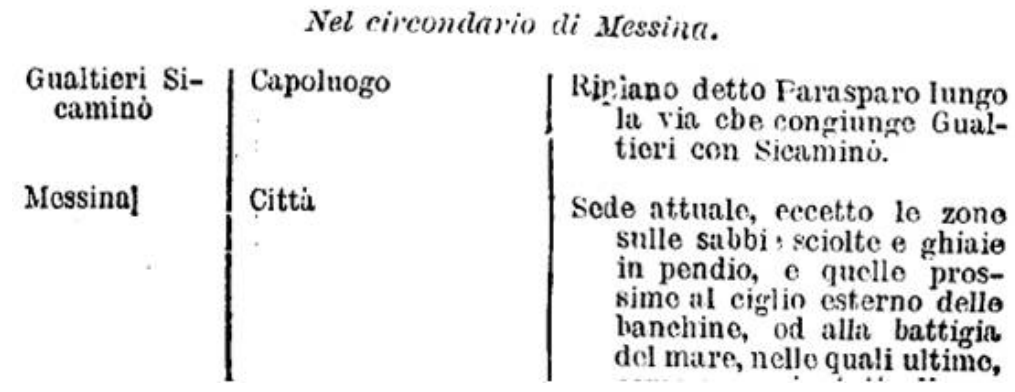

Fig. 4. Ricostruzione di Messina nella "sede attuale" (R.D. n. 542, 15 luglio 1909).

\section{Bibliografia}

Discussione ed approvazione del progetto di legge per l'applicazione del sistema di propulsione idropneumatica al piano inclinato dei Giovi (1870a), in Galletti G., Trompeo P. (a cura di). Atti del Parlamento subalpino, V legislatura - Sessione del 1853-54, vol. 8. Firenze: Botta, pp. 332-335.

Discussione generale del progetto di legge per l'applicazione di un nuovo sistema di propulsione idropneumatica al piano inclinato dei Giovi (1870b), in Galletti G., Trompeo P. (a cura di). Atti del Parlamento subalpino. Discussioni della Camera dei Deputati, V Legislatura - Sessione 1853-1854, vol. 5. Firenze: Botta, pp. 2067-2087.

Discussione sul progetto di legge per cui S.M. il Re Vittorio Emanuele II assume il titolo di Re d'Italia, in Resoconto della seduta del Senato del 26 febbraio 1861 [online]. URL: <http://www.senato.it/documenti/repository/notizie/2011/seduta_26febbraio18 61_senato.pdf $>$ [data di accesso: $31 / 01 / 2019$ ].

Leone M., Paoletti A., Robotti N. (2011). "I fisici e il Risorgimento". Il Nuovo Saggiatore, 27, pp. 30-42.

Matteucci C. (1861a). Relazione fatta al Senato il 24 febbraio 1861 dall'ufficio centrale, composto dai senatori De Gori, Giulini, Giorgini, Niutta, e Matteucci, relatore in Galletti G., Trompeo P. (a cura di) (1861). Atti del Parlamento italiano, Sessione del 1861, (VIII Legislatura), Documenti, $1^{\circ}$ periodo dal 18 febbraio al 23 luglio 1861. Torino: Botta, pp. 4-5.

Matteucci C. (1861b). Relazione fatta al Senato il 20 marzo 1861 dall'ufficio centrale composto dai senatori Plezza, Giulini, Marzucchi, Di Pollone, e Matteucci, relatore, in Galletti G., Trompeo P. (a cura di). Atti del Parlamento italiano, Sessione del 1861, (VIII Legislatura), Documenti, $1^{\circ}$ periodo dal 18 febbraio al 23 luglio 1861. Torino: Botta, p. 71.

Mosca C. (1869). Relazione fatta al Senato il 4 luglio 1854 dall'ufficio centrale, composto dai senatori Di Bagnolo, Di Collegno Giacinto, Giulio, Pallavicino-Mossi, 
e Mosca, relatore, in Galletti G., Trompeo P. (a cura di). Atti del Parlamento Subalpino, sessione del 1853-54. Documenti, vol. 2. Firenze: Botta, pp. 1347-1348.

Seguito della discussione di "Stati di previsione della spesa del Ministero dell'istruzione pubblica per gli esercizi finanziari 1921-22 e 1922-23” (n. 461) (1923), in Atti del Parlamento italiano, Camera dei Senatori, Legislatura XXVI, $1^{a}$ Sessione 19211923, Discussioni. Roma: Tipografia del Senato.

Svolgimento dell'interpellanza del senatore Del Giudice al ministro della pubblica istruzione (1923), in Atti del Parlamento italiano, Camera dei Senatori, Legislatura XXVI, $1^{a}$ Sessione 1921-1923, Discussioni. Roma: Tipografia del Senato.

Svolgimento dell'interpellanza del senatore Todaro al ministro dell'istruzione pubblica per sapere in che modo intenda il Governo ristabilire l'Università di Messina (1909), in Atti Parlamentari della Camera dei senatori. Discussioni. Legislatura XXIII, $1^{\circ}$ sessione 1909. Roma: Forzani, pp. 116-131.

\section{Fonti di archivio}

Roma:

Camera dei Deputati, Archivio della Camera regia,

- Disegni e proposte di legge e incarti delle commissioni (1848-1943), 308, 310 334 cc., vol. X.

- Disegni e proposte di legge e incarti delle commissioni (1848-1943), Minuta della Relazione dell'Ufficio centrale, c. 9 bis. 